\title{
Das Low-Budget-Wartezimmer-TV
}

\author{
Sie möchten Ihren Patienten schon im Wartezimmer einige Tipps zum Aufenthalt geben, \\ Ihnen Wissenswertes zu urologischen Krankheitsbildern und Neuigkeiten aus der Abteilung \\ oder Praxis zeigen? Eine Alternative zum professionellen Wartezimmer-TV stellt Ihnen unser \\ Autor in folgendem Tipp vor.
}

Professionelle Anbieter erstellen gegen Gebühr eine aufwändige, teilweise animierte Version des für Patienten gleichermaßen informativen wie unterhaltsamen Wartezimmer-TVs. Meist bestehen diese Angebote aus einem fixen Rahmenprogramm, in das individualisierte Angebote, Sprechzeiten, Fotos oder spezialisierte Leistungen eingebracht werden können. Dies ist ein kostenpflichtiger Service, der auch wegen der Kosten nicht in jedem Fall wahrgenommen wird.

Wir hatten die Idee eines eigenen, selbst erstellten Wartezimmer-TV-Programms, für das wir unsere Powerpoint-Kenntnisse genutzt haben. Vielleicht gerade wegen des eher einfachen aber individuellen Charakters findet dies großen Anklang: Die Vorstellung des Teams und die Geschichte der Abteilung wird ebenso referiert wie kurze, einfache Erklärungen zu klassischen urologischen Krankheitsbildern. Besondere Ereignisse wie der Auftritt der hiesigen Urologie bei einer Seniorenmesse mit eigenem Stand oder die Anschaffung der neuen ESWL werden von Patienten aufmerksam zur Kenntnis genommen. Auch nützliche Tipps für den stationären Aufenthalt (Visiten- und Essenszeiten, Lob- und Tadel-System, Angehörigen-Sprechstunde, Aussehen der Wahlleistungsstation) lassen sich so transportieren. Der in der urologischen Wartezone angebrachte Bildschirm mit einer SD-Karte ist so programmiert, dass

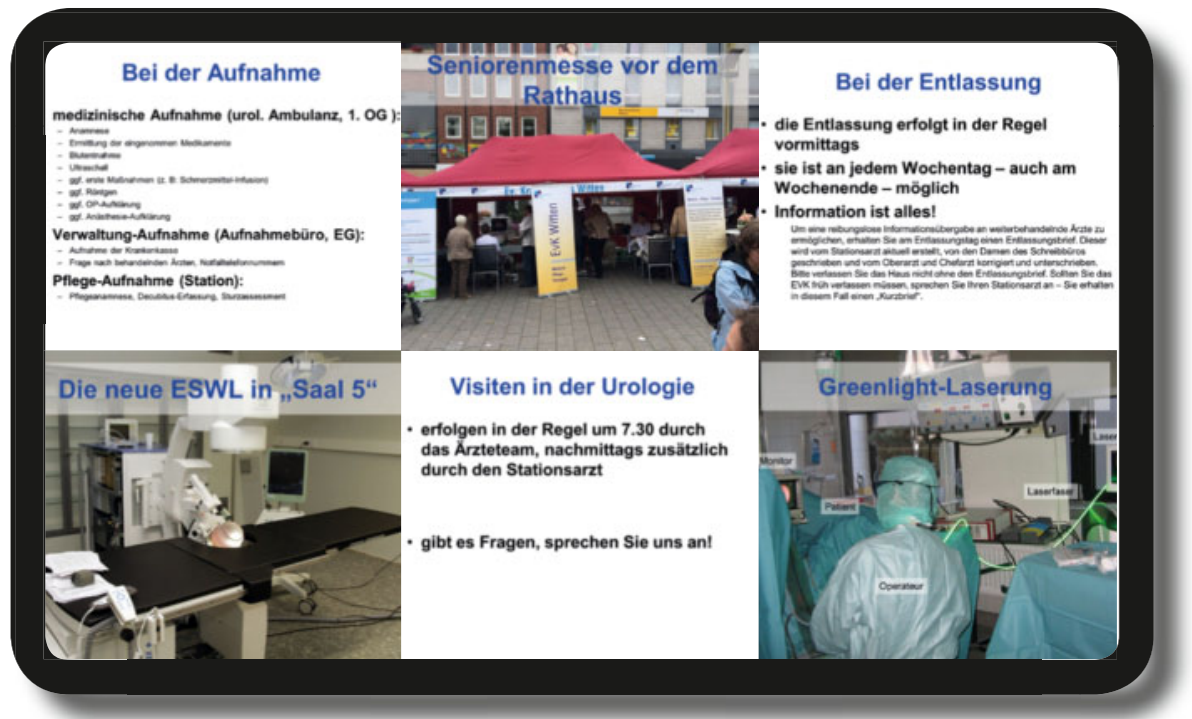

Beispielfolien für das Wittener Wartezimmer-TV: Informationen über Veranstaltungen, Organisatorisches sowie Standardbehandlungen - hier: ESWL und Greenlight-Laserung - ergeben einen unterhaltsamen und informativen Mix.

die Präsentation in einer Endlos-Schleife automatisch abläuft. Das Aktualisieren der Präsentation ist einfach und ohne Einschalten einer Fremdfirma möglich.

Die Vorteile der Patienteninformation, die Möglichkeit der Darstellung der Abteilung oder Praxis und nicht zuletzt die Aufklärung und Information der Patienten lassen bei niedrigen Kosten den überschaubaren Arbeitsaufwand vergessen.

\section{PD Dr. Andreas Wiedemann, Witten}

Korrespondenz:

PD Dr. Andreas Wiedemann

Urologische Klinik

Evangelisches Krankenhaus

im Diakoniewerk Ruhr gGmbH

Lehrstuhl für Geriatrie

der Universität Witten/Herdecke

Pferdebachstr. 27

58455 Witten

awiedemann@diakonie-ruhr.de

Interessenkonflikte:

Beratungstätigkeit: Dr. Pfleger, Pfizer; Vortragstätigkeit: Allergan, AMS Deutschland, Astellas, Berlin-Chemie, Janssen, Lilly Deutschland, Dr. Pfleger, Pfizer, PohlBoskamp; Studienfinanzierung: AMS Deutschland

\section{Thr Tipp in der Aktuellen Urologie}

Haben Sie einen interessanten Tipp oder Trick aus der Praxis für unsere Leser? Wir freuen uns über Ihre Manuskripte unter Aktuelle-Urologie@thieme.de! 